\title{
Spectroscopic study of formation, evolution and interaction of M31 and M33 with star clusters
}

\author{
Zhou Fan $^{1}$ and Yanbin Yang ${ }^{2}$ \\ ${ }^{1}$ Key Laboratory of Optical Astronomy, National Astronomical Observatories, Chinese \\ Academy of Sciences, 20A Datun Road, Chaoyang Dist., Beijing, China \\ email: zfan@bao.ac.cn \\ ${ }^{2}$ GEPI, Observatoire de Paris, CNRS, 5 Place Jules Janssen, Meudon F92195, France
}

\begin{abstract}
The recent studies show that the formation and evolution process of the nearby galaxies are still unclear. By using the Canada France Hawaii Telescope (CFHT) 3.6m telescope, the PanDAS shows complicated substructures (dwarf satellite galaxies, halo globular clusters, extended clusters, star streams, etc.) in the halo of M31 to $\sim 150 \mathrm{kpc}$ from the center of galaxy and M31-M33 interaction has been studied. In our work, we would like to investigate formation, evolution and interaction of M31 and M33, which are the nearest two spiral galaxies in Local Group. The star cluster systems of the two galaxies are good tracers to study the dynamics of the substructures and the interaction. Since 2010, the Xinglong $2.16 \mathrm{~m}$, Lijiang $2.4 \mathrm{~m}$ and MMT $6.5 \mathrm{~m}$ telescopes have been used for our spectroscopic observations. The radial velocities and Lick absorption-line indices can thus be measured with the spectroscopy and then ages, metallicities and masses of the star clusters can be fitted with the simple stellar population models. These parameters could be used as the input physical parameters for numerical simulations of M31-M33 interaction.
\end{abstract}

Keywords. galaxies: individual (M31) - galaxies: individual (M33) - galaxies: star clusters globular clusters: general - star clusters: general

\section{Introduction}

From 2008 to 2011, the Pan-Andromeda Archaeological Survey (PanDAS), which covers > $300 \mathrm{deg}^{2}$ M31-M33 field with CFHT 3.6m Telescope, was conducted by McConnachie et al. (2009). The survey discovered several new stellar stream features surrounding M31 and M33, respectively. It was found that dozens of dwarf galaxies and halo star clusters are distributed to the outer halo $\sim 150 \mathrm{kpc}$ from the center of M31. Observational evidences indicated that the structures may be due to M33 orbits around M31. The M31-M33 interaction was suggested by HI observations previously. Since the star clusters are much brighter than stars, the halo star clusters are good tracers to study the dynamics of the interactions.

\section{Observations and analysis}

Since 2010, we have started to observe the spectroscopy of the star clusters in the halo of M31 with the red channel spectrograph of $6.5 \mathrm{~m}$ MMT and BFOSC/OMR spectrograph of Xinglong 2.16m telescope. The star clusters of M31 were selected from RBC v.4 (http://www.bo.astro.it/M31; Galleti et al. 2004). The Lick absorption-line indices (see Worthey et al. 1994) of the spectroscopy were measured and fit with the stellar population model of Thomas et al. (2011), and then the ages, $[\mathrm{Fe} / \mathrm{H}]$ and $[\alpha / \mathrm{Fe}]$ can be derived, which provides the clues of the formation and evolution of the two galaxies. Meanwhile, 


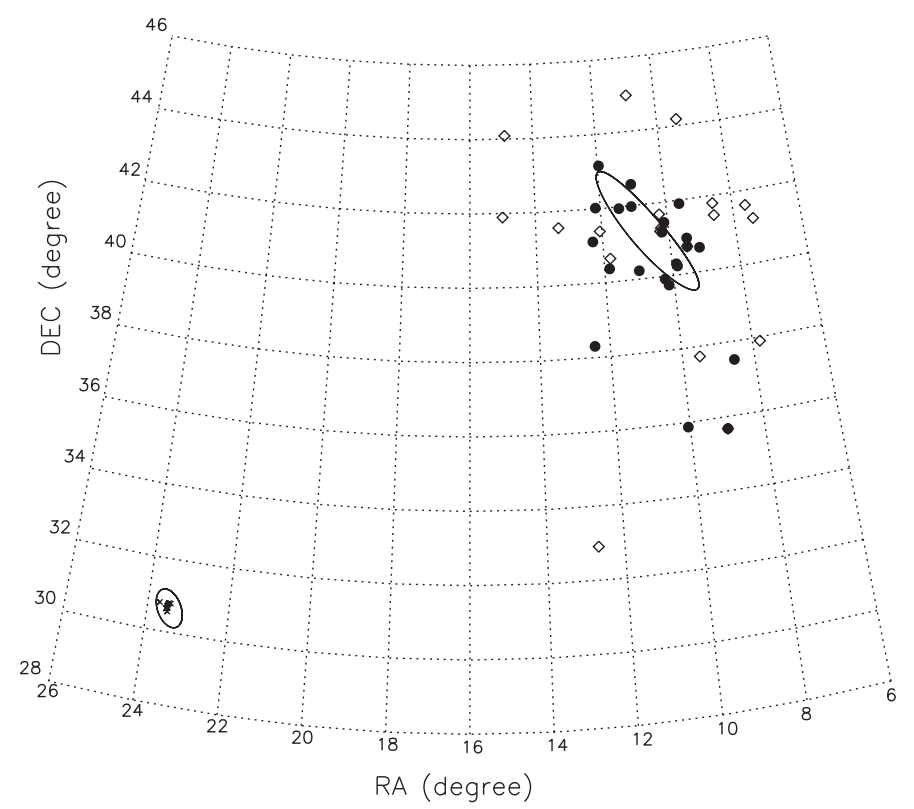

Figure 1. The distribution of star clusters that we have observed in M31 (larger ellipse) and M33 (smaller ellipse) field. The filled circles represent M31 star clusters observed with MMT; the open squares and crosses are star clusters of M31 and M33 observed with Xinglong 2.16m telescope, respectively.

the radial velocity $V_{r}$ can be estimated by fitting the absorption lines, which can be used to study the dynamics of the star cluster system as well as for tracing the dynamics of M31-M33 interaction.

Figure 1 shows the distribution of star clusters that we have observed so far with MMT and Xinglong 2.16m telescope. The open squares represent the M31 clusters observed with Xinglong 2.16m telescope and analyzed in Fan et al. (2011) and Fan et al. (2012). The filled circles are M31 clusters observed with MMT and the crosses show M33 star clusters observed with BFOSC spectrograph of Xinglong 2.16m telescope in Nov. 2014.

In the future, more spectroscopic observations are planed to be conducted with Lijiang $2.4 \mathrm{~m}$, and Guoshoujing (LAMOST) telescopes. The sample will be enlarged and more informations will be derived for the numerical simulations of the interaction of M31 and M33 galaxies.

\section{Acknowledgements}

This research is supported by the National Natural Science Foundation of China Grant No. 11373003 and National Key Basic Research Program of China (973 Program) No. 2015 CB857002.

\section{References}

Fan, Z., Huang, Y. F., Li, J. Z., Zhou, X., Ma, J., et al. 2011, RAA, 11, 1298

Fan, Z., Huang, Y. F., Li, J. Z., Zhou, X., Ma, J., \& Zhao, Y. H. 2012, RAA, 12, 829

Galleti, S., Federici, L., Bellazzini, M., Fusi Pecci, F., \& Macrina, S. 2004, A\&\&A, 416, 917

McConnachie, A. W., et al. 2009, Nature, 461, 66

Perina, S., Cohen, J. G., Barmby, P., et al. 2010, A\& $A$, 511, A23

Thomas, D., Maraston, C., \& Johansson, J. 2011, MNRAS, 412, 2183

Worthey, G., Faber, S. M., Gonzalez, J. J., \& Burstein, D. 1994, ApJS, 94, 687 